\title{
Allergen-Specific Immunotherapy in Asthma
}

Marek Jutel, $M D^{1,2, *}$

\author{
Address \\ ${ }^{1 *}$ Department of Clinical Immunology, Wrocław Medical University, \\ Wroclaw, Poland \\ Email: marek.jutel@umed.wroc.pl \\ ${ }^{2}$ ALL-MED Medical Research Institute, Wroclaw, Poland
}

Published online: 12 March 2014

(C) The Author(s) 2014. This article is published with open access at Springerlink.com

Keywords Asthma - Allergen-specific immunotherapy - SLIT • SCIT • Bronchodilators • Long acting bronchodilators • LABA · Inhaled corticosteroids · Omalizumab

\section{Opinion statement}

Current asthma therapies can effectively control symptoms and the on-going inflammatory process; however, they do not affect the underlying, dysregulated immune response. Thus, they are limited to blunting the progression of the disease, which relapses on ceasing the treatment. Allergen-specific immunotherapy (AIT) is the only etiology-based treatment capable of disease modification. Recent evidence provided a plausible explanation for its multiple mechanisms inducing both rapid desensitization and long-term allergen-specific immune tolerance, as well as the suppression of allergic inflammation in the affected tissues. Although the current guideline documents give both subcutaneous (SCIT) and sublingual (SLIT) immunotherapy a conditional recommendation in allergic asthma due to the moderate and low quality of evidence, respectively, a growing body of evidence from doubleblind, placebo-controlled studies shows that both SLIT and SCIT are effective in reducing symptom scores and medication use, improving quality of life, and inducing favorable changes in specific immunologic markers. Due to the very limited evidence from head-to-head comparative studies and variability of the end-point used in different studies, it is currently not possible to assess superiority of either route of vaccine administration.

\section{Introduction}

Asthma is a chronic inflammatory disease with high incidence, about 300 million people worldwide. The pathological process of the airways is associated with hyperresponsiveness, which leads to recurrent episodes of wheezing, dyspnoea, chest tightness and cough, as well as variable airflow obstruction that may become permanent due to airway remodelling [1]. Asthma is not exclusively associated with allergy/ atopy. However, more than $50 \%$ of the asthmatic population is allergic/atopic, but only a fraction of al- 
lergic subjects develop asthma. Thus, the pathophysiology of asthma is very complex and includes several disease variants [2]. Distinct phenotypes of asthma describe clinical and morphologic characteristics as well as unique responses to treatment. In addition, various endotypes have been described that define intrinsically distinct pathogenetic mechanisms [2]. For a long time, asthma has been considered mainly a $\mathrm{T}_{\mathrm{h}} 2$ cell-mediated disorder with interleukin (IL)-4, IL-13, IL-9 and IL-5 involved in the airway inflammation [3]. However, many other cell types including Treg, $\mathrm{T}_{\mathrm{h}} 1, \mathrm{~T}_{\mathrm{h}} 17$, natural killer (NK) and $\beta \beta$ T cells are also involved $[2,4$, 5]. It is assumed that the more severe asthma symptoms develop, the more $T_{h} 1$ and $T_{h} 17$ cells are involved. In particular, neutrophilic infiltration and inflammation triggered by the production of tumor necrosis factor (TNF) $\beta$, IL-17, and IL-27 might account for corticosteroid resistance [6]. Thus, endotyping asthma based on disease mechanisms could eventually lead to individualized management.

Current asthma therapies can effectively control symptoms and the on-going inflammatory process; however, they do not affect the underlying, dysregulated immune response. Thus, they are limited to blunting the progression of the disease, which relapses on ceasing the treatment.

Bronchodilators are effective in reducing airway obstruction. Current guidelines recommend combined use of long-acting bronchodilators (LABA) with inhaled corticosteroids (ICS) due to safety concerns [7]. Alternative classes of bronchodilators, such as vasoactive intestinal peptide analogs and potassiumchannel openers, are currently under investigation. ICS are currently basic controllers in asthma therapy. These 'conventional' anti-asthmatic agents are constantly updated both for the new molecules as well as more convenient and effective delivery devices, which also improve patient compliance. The major setbacks with the usage of ICS and LABA include the fear of long-term side effects, compliance with inhaled administration, as well as the relapse of symptoms after discontinuation of drug administration. In a considerable number of patients, acceptable symptom control is not achieved with these drug classes. Thus, considering the complexity of asthma pathogenesis, therapies aiming at blocking critical effector molecules are under intensive investigation [8]. Currently, omalizumab is the only anti-IgE monoclonal antibody approved for asthma treatment [9]. In addition, novel drugs utilizing immune-modulatory mechanisms including suppression of disease-associated cytokines are being developed (see this issue, Akdis M. et al.).

Allergen-specific immunotherapy (AIT) is the only etiology-based treatment capable of disease modification, as demonstrated by prevention of both the onset of new allergic sensitizations and disease progression.

Due to its disease-modifying effects based on its immunomodulatory properties, AIT is the only real curative modality in allergic asthma $[10 \bullet \bullet]$.

Recent evidence has provided a plausible explanation for the multiple mechanisms of AIT, which induce both rapid desensitization and long-term allergen-specific immune tolerance, as well as the suppression of allergic inflammation in the affected tissues. The described mechanisms include changes in the profile of allergenspecific memory $\mathrm{T}$ - and B-cell responses, the synthesis of specific antibody isotypes that skew the immune response towards a non-inflammatory pattern, as well as decreased activation, tissue migration, and degranulation of effector cells including mast cells, basophils, and eosinophils [11]. These findings, together with the new biotechnological approaches, create a platform for development of the advanced vaccines. Moreover, reliable biomarkers could be selected and validated with the intention to select the patients who will benefit most from this immune-modifying treatment. Thus, AIT could provide a complete cure for a larger number of allergic patients [12].

\section{Treatment}

AIT involves the repeated administration of allergen preparations in order to induce clinical and immunologic tolerance to the offending allergen. The two most commonly prescribed routes for AIT are subcutaneous (SCIT) and sublingual (SLIT). The sublingual route has emerged as an effective and safer alternative to subcutaneous administration. Factors 
considered for the selection of route include vaccine availability or approval, geographic location, cost, and the patient's characteristics or the

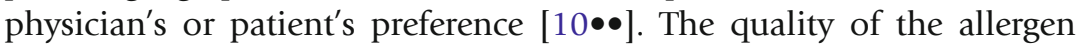
preparations used for AIT has been constantly improved. In addition, novel vaccines are being developed by using novel adjuvants, or changing the allergen to reduce allergenic activity, increase immunogenicity. Cloning of allergen proteins with use of recombinant DNA technology enabled the production of vaccines that have well defined molecular, immunologic, and biological characteristics. Genetic engineering enables modifications of the molecular structure of allergens [13].

Both SCIT and SLIT are of proven value in asthma. Numerous doubleblind, placebo-controlled trials have confirmed that SLIT and SCIT are effective in reducing symptom scores and medication use, improving quality of life in asthma, and inducing favorable changes in specific immunologic markers $[10 \bullet \bullet, 12]$. Overall, moderate-to-high (somewhat weaker in children) evidence was found for the efficacy and safety of both SCIT and SLIT for the treatment of allergic asthma, and it has not been possible to assess superiority of either route over the other $[14 \bullet \bullet, 15 \bullet \bullet]$.

However, the current documents of ARIA (Allergic Rhinitis and its Impact on Asthma) $[16,17]$ give both SCIT and SLIT a conditional recommendation in allergic asthma due to moderate and low quality of evidence, respectively. According to the GINA (Global Initiative for Asthma) report updated in 2012, AIT should be considered only after strict environmental avoidance and pharmacologic intervention, including ICS [17].The evidence for SCIT efficacy has been analyzed in the Cochrane review, which reported an overall clinical efficacy; that is, reductions in asthma symptom scores, medication usage, and allergen-specific bronchial hyperreactivity (BHR), and limited reduction in non-specific BHR [18]. The numbers of patients needed to treat in order to avoid asthma symptom deterioration or increase in medications were estimated as three and five, respectively. The effects on lung function were not consistent among trials. Also, more recent studies on efficacy of subcutaneous immunotherapy in asthma show similar treatment effect [19•, 20]. The most recent meta-analysis of the effectiveness of SCIT in the treatment of allergic rhinitis and asthma up to May 2013 concluded that SCIT reduces asthma symptoms and asthma medication usage. Respiratory adverse reactions to SCIT are common, but no deaths were reported in the included studies [21].

For the sublingual route of administration, most of the published evidence comes from studies primarily in rhinitis patients [22-26]. Thus, the studies are often not adequately powered for a definite conclusion. In addition, no consensus exists on the optimal endpoints. The efficacy of SLIT in seasonal allergy is now well documented both in adults and children. The data for perennial allergies is less convincing, particularly in children. In a large study including 602 asthmatic patients allergic to house dust mites (HDM), a reduced need for ICS for asthma control was demonstrated compared with placebo after only 1 year of treatment [27].

Recent systematic reviews graded the evidence for the effectiveness of SCIT and SLIT according to recommendations of the Grading of Recommendations Assessment, Development and Evaluation Working Group [14••]. In their review, Lin and colleagues point out that eight of 13 studies reported 
greater than $40 \%$ improvement versus the comparator (placebo, pharmacotherapy, or other SLIT regimens) [Lin, \#425, 15, 28].

In a review limited to a pediatric population receiving SCIT, SLIT, or usual care, it was concluded that SCIT reduces symptoms and medication scores,

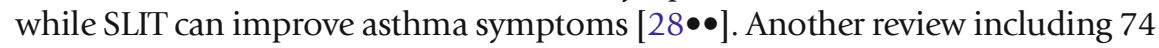
references on SCIT and 60 on SLIT and eight comparative (SCIT versus SLIT) studies showed similar tendencies. A potential steroid-sparing effect of AIT is of

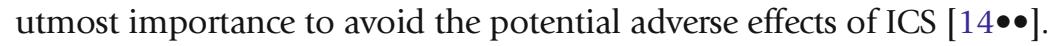

In a recent study, it has been demonstrated that after 3 years of SLIT (birch pollen) in adult patients inadequately responding to a low dose of ICS, a significantly better control can be achieved by adding SLIT for 12 weeks [25]. In the 'real-life' retrospective study of Trebuchon et al., $63 \%$ of patients with asthma due to sensitization to HDM who received SLIT showed improved symptoms and a reduction in medication. However, further studies specifically designed to address the effect of AIT in asthma are needed [29].

In particular, the ongoing phase III confirmatory, double-blind, placebocontrolled trials with both SCIT (Roxall Medizin, Allergopharma) and SLIT (ALK, Stallergen) in perennial HDM allergy will provide more solid evidence of the efficacy of AIT in asthma (data from ClinicalTrials.gov, EU Clinical Trials Register, Japan Pharmaceutical Information Center: Clinical Trials Information) [27].

\section{Contraindications and side effects}

Along with general contraindications, severe or uncontrolled asthma is the most important and independent risk factor for both nonfatal and fatal adverse reactions to SCIT $[10 \bullet \bullet]$.

A Cochrane systematic review [18] showed that the possibility of local or systemic adverse effects due to SCIT must be considered. If 16 patients are treated with SCIT, one would be expected to develop a local adverse reaction, and if nine patients are treated, one would be expected to develop a systemic reaction of any grade of severity. Thus, patients should be observed typically for 30-45 minutes after injection to assure proper management of systemic reactions. [30]. SLIT has been shown to be safer than SCIT and so far no fatalities have been reported. The side effects of SLIT are mainly local. In total, 11 cases of anaphylaxis were reported during SLIT but asthma was not considered a possible risk factor. Nevertheless, SLIT is not recommended to be administered in uncontrolled disease [31•].

\section{Dosing}

For many allergens, effective SLIT or SCIT doses have not been established. With grass pollen, the effective cumulative SLIT doses appear to be as high as 20 to 30 times greater than the effective SCIT doses.

Multiallergen SLIT has not been well studied, and its use might be limited by the increased cost of allergen extracts and the inconvenience of taking multiple doses. 
The comparative effect of precoseasonal and continuous grass pollen SLIT in children has been investigated by Stelmach et al. [32] in a 2-year, prospective, randomized, double-blind, placebo-controlled trial. Both precoseasonal and continuous regimens were similarly associated with a substantial reduction in the combined symptoms/medication score, including the asthma score, when compared with placebo.

Duration of treatment

The optimal duration for an AIT course is still a matter of debate, especially for SLIT. A recent study in asthmatic children showed that 3 years of SCIT is an adequate duration for the treatment of asthma in HDM-allergic subjects [19•].

\section{Cost/cost effectiveness}

Studies comparing cost effectiveness between patients treated for 3 years with AIT versus those treated with pharmacotherapy alone have indicated that AIT might be associated with cost savings as high as $80 \% 3$ years after completion of treatment $[10 \bullet \bullet]$.

Emerging therapies

The most promising novel approaches with phase II and III clinical studies available or on the way include

- novel adjuvants (MPL, MAT technology)

- modified allergen molecules (further development of allergoids)

- peptides, recombinant allergens (birch, grass pollen, cat dander)

- new routes of AIT (e.g., intralymphatic, epicutaneous)

\section{Pediatric considerations}

No pediatric meta-analyses are available for SCIT. A more recent metaanalysis of SLIT in children reported a moderate effectiveness on asthma symptoms and medication intake [33]. However, a number of studies are characterized by shortcomings in sample size and methodology [34, 35]. New well controlled studies are postulated by the European Medicines Agency (EMA) within the Paediatric Investigation Plan (PIP).

\section{Compliance with Ethics Guidelines}

\section{Conflict of Interest}

Marek Jutel is a consultant to Anergis SA (CH), Allergopharma (Ger), Biomay (A) and received honoraria for lecture fees from GSK, Allergopharma, Stallergens, ALK.

Human and Animal Rights and Informed Consent

This article does not contain any studies with human or animal subjects performed by any of the authors. 


\section{Open Access}

This article is distributed under the terms of the Creative Commons Attribution License which permits any use, distribution, and reproduction in any medium, provided the original author(s) and the source are credited.

\section{References and Recommended Reading}

Papers of particular interest, published recently, have been highlighted as:

- Of importance

- Of major importance

1. Bateman ED, Hurd SS, Barnes PJ, Bousquet J, Drazen JM, FitzGerald M, et al. Global strategy for asthma management and prevention: GINA executive summary. Eur Respir J. 2008;31(1):143-78.

2. Agache I, Akdis C, Jutel M, Virchow JC. Untangling asthma phenotypes and endotypes. Allergy. 2012;67(7):835-46.

3. Akdis CA. Therapies for allergic inflammation: refining strategies to induce tolerance. Nat Med. 2012;18(5):736-49.

4. Kaczorowski M, Jutel M. Human T regulatory cells: on the way to cognition. Arch Immunol Ther Exp (Warsz). 2013;61(3):229-36.

5. Jutel M, Akdis CA. T-cell subset regulation in atopy. Curr Allergy Asthma Rep. 2011;11(2):139-45.

6. Wang W, Li JJ, Foster PS, Hansbro PM, Yang M. Potential therapeutic targets for steroid-resistant asthma. Curr Drug Targets. 2010;11(8):957-70.

7. Jaeschke R, O'Byrne PM, Mejza F, Nair P, Lesniak W, Brozek J, et al. The safety of long-acting beta-agonists among patients with asthma using inhaled corticosteroids: systematic review and metaanalysis. Am J Respir Crit Care Med. 2008;178(10):1009-16.

8. Barnes PJ. New therapies for asthma: is there any progress? Trends Pharmacol Sci. 2010;31(7):335-43.

9. Kopp MV. Omalizumab: anti-IgE therapy in allergy. Curr Allergy Asthma Rep. 2011;11(2):101-6.

10.• Burks AW, Calderon MA, Casale T, Cox L, Demoly P, Jutel M, et al. Update on allergy immunotherapy: American Academy of Allergy, Asthma \& Immunology/European Academy of Allergy and Clinical Immunology/PRACTALL consensus report. J Allergy Clin Immunol. 2013;131(5):1288-96 e1283.

Comprehensive report of the AAAAI and EAACI consensus on AIT.

11. Jutel M, Van de Veen W, Agache I, Azkur KA, Akdis $\mathrm{M}$, Akdis CA. Mechanisms of allergen-specific immunotherapy and novel ways for vaccine development. Allergol Int. 2013;62(4):425-33.

12. Calderon MA, Casale T, Cox L, Akdis CA, Burks AW, Nelson HS, et al. Allergen immunotherapy: a new semantic framework from the European Academy of Allergy and Clinical Immunology/ American Academy of Allergy, Asthma and Im-
munology/PRACTALL consensus report. Allergy. 2013;68(7):825-8.

13. Jutel M, Solarewicz-Madejek K, Smolinska S. Recombinant allergens: the present and the future. Hum Vaccin Immunother. 2012;8(10):1534-43.

14.• Lin SY, Erekosima N, Suarez-Cuervo C, Ramanathan M, Kim JM, Ward D, et al. Agency for healthcare research and quality (US); 2013 Mar. Report No.: 13-EHC061EF. AHRQ Comparative Effectiveness Reviews; 2013.

Updated systematic review providing a overview of efficacy of SLIT and SCIT including asthma.

15.• Lin SY, Erekosima N, Kim JM, Ramanathan M, Suarez-Cuervo C, Chelladurai Y, et al. Sublingual immunotherapy for the treatment of allergic rhinoconjunctivitis and asthma: a systematic review. JAMA. 2013;309(12):1278-88.

Systematic review on the efficacy of SLIT in the treatment of allergic rhinitis and asthma, also addressing optimal dosing strategies.

16. Bousquet J, Schunemann HJ, Samolinski B, Demoly $\mathrm{P}$, Baena-Cagnani CE, Bachert $\mathrm{C}$, et al. Allergic Rhinitis and its Impact on Asthma (ARIA): achievements in 10 years and future needs. J Allergy Clin Immunol. 2012;130(5):1049-62.

17. Brozek JL, Bousquet J, Baena-Cagnani CE, Bonini S, Canonica GW, Casale TB, et al. Allergic Rhinitis and its Impact on Asthma (ARIA) guidelines: 2010 revision. J Allergy Clin Immunol. 2010;126(3):466-76.

18. Abramson MJ, Puy RM, Weiner JM. Injection allergen immunotherapy for asthma. Cochrane Database Syst Rev. 2010;(8):CD001186.

19. Stelmach I, Sobocinska A, Majak P, Smejda K, Jerzynska J, Stelmach W. Comparison of the longterm efficacy of 3- and 5-year house dust mite allergen immunotherapy. Ann Allergy Asthma Immunol. 2012;109(4):274-8.

Interesting study providing evidence on the optimal duration of AIT.

20. Yukselen A, Kendirli SG, Yilmaz M, Altintas DU, Karakoc GB. Effect of one-year subcutaneous and sublingual immunotherapy on clinical and laboratory parameters in children with rhinitis and asthma: a randomized, placebo-controlled, double-blind, double-dummy study. Int Arch Allergy Immunol. 2012;157(3):288-98. 
21. Erekosima N, Suarez-Cuervo C, Ramanathan M, Kim JM, Chelladurai Y, Segal JB, et al. Effectiveness of subcutaneous immunotherapy for allergic rhinoconjunctivitis and asthma: a systematic review. Laryngoscope. 2013. doi:10.1002/lary.24295.

22. De Castro G, Zicari AM, Indinnimeo L, Tancredi G, di Coste A, Occasi F, et al. Efficacy of sublingual specific immunotherapy on allergic asthma and rhinitis in children's real life. Eur Rev Med Pharmacol Sci. 2013; 17(16):2225-31.

23. Peng W, Liu E. Factors influencing the response to specific immunotherapy for asthma in children aged 5-16 years. Pediatr Int. 2013;55(6):680-4.

24. Rosewich M, Arendt S, El Moussaoui S, Schulze J, Schubert R, Zielen S. Bronchial allergen provocation: a useful method to assess the efficacy of specific immunotherapy in children. Pediatr Allergy Immunol. 2013;24(5):434-40.

25. Marogna M, Braidi C, Bruno ME, Colombo C, Colombo F, Massolo A, et al. The contribution of sublingual immunotherapy to the achievement of control in birch-related mild persistent asthma: a real-life randomised trial. Allergol Immunopathol (Madr). 2012;41(4):216-24.

26. Wahn U, Klimek L, Ploszczuk A, Adelt T, Sandner B, Trebas-Pietras E, et al. High-dose sublingual immunotherapy with single-dose aqueous grass pollen extract in children is effective and safe: a double-blind, placebo-controlled study. J Allergy Clin Immunol. 2012;130(4):886-93 e885.

27. Mosbech H. Tolerability and efficacy of house dust mite AIT. Allergy. 2011;66 Suppl 95:55-6.

28.• Kim JM, Lin SY, Suarez-Cuervo C, Chelladurai Y, Ramanathan M, Segal JB, et al. Allergen-specific immunotherapy for pediatric asthma and rhinoconjunctivitis: a systematic review. Pediatrics. 2013;131(6):1155-67.
Comprehensive systematic review of AIT in the pediatric population.

29. Trebuchon F, David M, Demoly P. Medical management and sublingual immunotherapy practices in patients with house dust mite-induced respiratory allergy: a retrospective, observational study. Int J Immunopathol Pharmacol. 2012;25(1):193206.

30. Cox L, Nelson H, Lockey R, Calabria C, Chacko T, Finegold I, et al. Allergen immunotherapy: a practice parameter third update. J Allergy Clin Immunol. 2011;127(1 Suppl):S1-55.

31. Calderon MA, Simons FE, Malling HJ, Lockey RF, Moingeon P, Demoly P. Sublingual allergen immunotherapy: mode of action and its relationship with the safety profile. Allergy. 2012;67(3):302-11.

Comprehensive report addressing the issue of safety of AIT

32. Stelmach I, Kaluzinska-Parzyszek I, Jerzynska J, Stelmach P, Stelmach W, Majak P. Comparative effect of pre-coseasonal and continuous grass sublingual immunotherapy in children. Allergy. 2011;67(3):312-20.

33. Penagos M, Passalacqua G, Compalati E, BaenaCagnani CE, Orozco S, Pedroza A, et al. Metaanalysis of the efficacy of sublingual immunotherapy in the treatment of allergic asthma in pediatric patients, 3 to 18 years of age. Chest. 2008;133(3):599-609.

34. Nieto A, Mazon A, Pamies R, Bruno L, Navarro M, Montanes A. Sublingual immunotherapy for allergic respiratory diseases: an evaluation of meta-analyses. J Allergy Clin Immunol. 2009;124(1):157-61. e151132.

35. Compalati E, Penagos M, Tarantini F, Passalacqua G, Canonica GW. Specific immunotherapy for respiratory allergy: state of the art according to current metaanalyses. Ann Allergy Asthma Immunol. 2009;102(1):22-8. 